"This is a post-peer-review, pre-copyedit version of an article published in Applied Physics A. The final authenticated version is available online at: http://dx.doi.org/10.1007/s00339-016-0725-0 and https://link.springer.com/article/10.1007/s00339-016-0725-0 ".

\title{
Dual phylogenetic staining protocol for simultaneous analysis of yeast and bacteria in artworks
}

Marina González-Pérez ${ }^{1}$, Catarina Brinco ${ }^{1}$, Ricardo Vieira ${ }^{1}$, Tânia Rosado ${ }^{1}$, Guilhem Mauran $^{1,2,3}$, António Pereira ${ }^{1,4}$, António Candeias ${ }^{1,4}$, Ana Teresa Caldeira ${ }^{1,4 *}$

1 HERCULES Laboratory, Évora University, Largo Marquês de Marialva 8, 7000809, Évora (Portugal), +351 266740 800, atc@uevora.pt

2 ARCHMAT Erasmus Mundus Master in Archaeological Materials Science, Évora University, Largo Marquês de Marialva 8, 7000-809 Évora, Portugal

3 Département de Préhistoire du Museum National d'Histoire Naturelle, UMR 7194 CNRS-MNHN-UPVD, Sorbonne Université, Musée de l'Homme, 17 Place du Trocadéro, 75116 Paris, France

4 Chemistry Department, School of Sciences and Technology, Évora University, Rua Romão Ramalho 59 7000-671, Évora (Portugal)

* atc@uevora.pt, +351266740800

\section{Acknowledgments}

The authors thank "HIT3CH - HERCULES Interface for Technology Transfer and Teaming in Cultural Heritage", Ref: ALT20-03-0246-FEDER-000004, co-funded by the Regional Development European Fund through ALENTEJO 2020 (Programa Operacional Regional do Alentejo). This work was funded by National Funds through FCT - Fundação para a Ciência e a Tecnologia: project "MICROTECH-ART Microorganisms Thriving on and Endamaging Cultural Heritage -an Analytical Rapid Tool-" (PTDC/BBB-IMG/0046/2014), post-doctoral grant SFRH/BPD/100754/2014 and BSc fellowship microtech_lic_2016.

Authors are also grateful to the Alentejo Regional Directorate for Culture and archaeologist António Carlos Silva for allowing the sampling from Escoural Cave. 


\section{Abstract}

The detection and analysis of metabolically active microorganisms are useful to determine those directly involved in the biodeterioration of Cultural Heritage $(\mathrm{CH})$. Fluorescence In Situ Hybridization with oligonucleotide probes targeted at rRNA (RNAFISH) has demonstrated to be a powerful tool for signaling them. However, more efforts are required for the technique to become a vital tool for the analysis of CH's microbiological communities.

Simultaneous analysis of microorganisms belonging to different kingdoms, by RNAFISH in-suspension approach, could represent an important progress: it could open the door for the future use of the technique to analyze the microbial communities by flow cytometry, which has shown to be a potent tool in environmental microbiology.

Thus, in this work various already implemented in-suspension RNA-FISH protocols for ex situ analysis of yeast and bacteria were investigated and adapted for allowing the simultaneous detection of these type of microorganisms. A deep investigation of the factors that can affect the results was carried out, focusing particular attention on the selection of the fluorochromes used for labelling the probe set. The resultant protocol, involving the use of EUK516-6-FAM/EUB338-Cy3 probes combination, was validated using artificial consortia and gave positive preliminary results when applied in samples from a real case study: the Paleolithic archaeological site of Escoural Cave (Alentejo, Portugal). This approach represents the first dual staining RNA-FISH in-suspension protocol developed and applied for the simultaneous investigation of $\mathrm{CH}$ biodeteriogenic agents belonging to different kingdoms.

Keywords: Fluorescence In Situ Hybridization; RNA-FISH; Biodeterioration; Cultural Heritage Microbiology; Phylogenetic staining.

\section{Introduction}

Biodeteriogenic microorganisms are one of the major threats to Tangible Cultural Heritage $(\mathrm{CH})$ preservation and conservation [1,2]. For addressing this problem, it is crucial to have access to straightforward tools that allow to target them [1,2]. Thus, it is of utmost importance to continuously develop and improve non-culture-based techniques that allow to detect and analyze the viable microorganisms thriving in $\mathrm{CH}$ assets [1-3]. RNA-Fluorescence In Situ Hybridization (RNA-FISH) has been already applied to this end $[1,2]$. Its application has not only provided a snapshot of the identity, presence and abundance of the microorganisms involved in the biodeterioration of $\mathrm{CH}$ objects [4-12], 
but has also led to the discovery of novel microorganisms thriving on them [13]. However, in spite of the already-demonstrated analytical potential $[11,14]$ and versatility of RNA-FISH technique for unveiling potential biodeteriogens of $\mathrm{CH}$ [7-10,15-22], it continues to be scarcely applied in this field.

This technique relies on turning the target cells into fluorescent cells, while maintaining their integrity [23]. This is achieved through hybridization of fluorescently labeled oligonucleotide probes (RNA-FISH probes) to the target complementary RNA sequence within the cells [23].

The RNA-FISH protocols already applied for investigating biodeterioration of $\mathrm{CH}$ has been performed in situ (directly in the material) [ 10,16,17] or ex situ (in coated-slides or tape-strip)[7-9,16-22] for investigating biodeterioration of a wide variety of materials (stone, rock, glass, mats, metals, mortars, polymers, wood, among others)[7-10,15-22], employing almost exclusively paraformaldehyde as fixative and analyzing the cells by epifluorescence microscopy or Confocal Laser Scanning Microscopy (CLSM).

The introduction of: i) novel FISH probes for detecting specific microorganisms thriving in $\mathrm{CH}[14,19]$; ii) multi-probe sets for enhancing the signal [11]; and iii) the tapestrip sampling method [24], are some examples of the improvements already included in the RNA-FISH protocols applied for assessing the microbial colonizers of $\mathrm{CH}$ objects/assets. Their implementation has allowed to overcome some of the limitations of the technique (e.g. autofluorescence interference from microorganisms or from inorganic surrounding material and low intense probe-conferred signals due to low cell permeability or low ribosome content[2,23]). However, there is still much to be done.

Thus, our work is focused on developing new strategies for facing the experimental difficulties found in the application of RNA-FISH in CH microbiology and for exploiting its analytical potential in this field [25-27]. The single staining in-suspension protocols previously developed by us for the ex situ analysis of bacteria and fungi isolates from $\mathrm{CH}$ materials allow to: i) avoid the use of paraformaldehyde (associated to fixation-induced fluorescence [28]); ii) reduce/eliminate background autofluorescence; and iii) obtain intense FISH signals using universal Cy3-labeled probes both by microscopy or flow cytometry analysis [26,27].

There is a need for cheaper and faster in-suspension dual phylogenetic staining approaches. They allow to analyze simultaneously two target microorganisms, reducing by half the number of assays required to assess the composition of microbial communities while opening the door for their analysis by flow cytometry. This powerful automatic and 
accurate tool is already being extensively exploited for analyzing the microbial communities in environmental samples [29].

Thus, the aim of this work was to develop a dual staining in-suspension RNA-FISH protocol for simultaneous ex situ detection of Eukarya and Eubacteria thriving in $\mathrm{CH}$. Its application in $\mathrm{CH}$ microbiology will allow to reduce the amount of sample and the number of analysis required for microscopic detection while making the implementation of Flow-FISH in $\mathrm{CH}$ microbiology one step closer.

\section{Materials and methods}

\subsection{Samples}

\subsubsection{Microorganisms}

Two model microorganisms were selected for pure culture and artificial consortium experiments: the model bacterium Escherichia coli ATCC 25922 belonging to the American Type Culture Collection (Manassas, VA, USA) and the model yeast Saccharomyces cerevisiae CCMI 396 from the Culture Collection of Industrial Microorganisms (Lisbon). The cells were maintained at $4^{\circ} \mathrm{C}$ in NA and YPD-Agar slants, respectively. The Erlenmeyer flasks $(250 \mathrm{~mL})$ containing $100 \mathrm{~mL}$ of NB and YEPD were inoculated and incubated at $30^{\circ} \mathrm{C}$ under continuous shaking at $120 \mathrm{rpm}$. The cells were harvested from cultures in the latency, exponential or stationary growth phases $(1,5$ or $24 \mathrm{~h}$ after inoculation). Aliquots of the culture $(20.0 \mathrm{~mL})$ were collected in $50 \mathrm{~mL}$ screwcap centrifuge tubes and washed with Phosphate Buffered Saline (PBS, $130.0 \mathrm{mM} \mathrm{NaCl}$, 8.0 $\mathrm{mM} \mathrm{NaH}_{2} \mathrm{PO}_{4}, 2.7 \mathrm{mM} \mathrm{KCl}, 1.5 \mathrm{mM} \mathrm{KH}_{2} \mathrm{PO}_{4}, \mathrm{pH}$ 7.2).

\subsubsection{Real samples}

Various microsamples were taken from the Escoural Cave (Montemor-o-Novo, Alentejo, Portugal) that is the only cavity discovered in Portugal where Upper Paleolithic rock art has been identified. They were collected, ensuring the representativeness, from areas of the walls' surface with visible microbial proliferation by scratching them with a scalpel. They were stored in sterile Petri dishes at $4^{\circ} \mathrm{C}$ for their transport till their analysis (within $24 \mathrm{~h}$ ).

Two micro-samples, GdE 1 and GdE 2, were analyzed by RNA-FISH. The samples were transferred to $50 \mathrm{~mL}$ screw-cap centrifuge tubes, 2,0 $\mathrm{ml}$ of Maximum Recovery Diluent (MRD, Sigma-Aldrich,) were added and maintained overnight at $30.0^{\circ} \mathrm{C}$ with 
continuous shaking (140 rpm). The resultant cellular suspensions were processed as described in sections 2.2.4, 2.3 and 2.4.

\subsection{RNA-FISH analysis}

\subsubsection{RNA-FISH probes}

The following probes labelled with $\mathrm{Cy} 3, \mathrm{Cy} 5$ or 6-FAM dyes at the 5'end were used: i) EUB338 (5'-GCTGCCTCCCGTAGGAGT-3') for analyzing E. coli, in isolates and synthetic consortium experiments, and most bacteria in microbial suspensions from Cultural Heritage samples [30]; and ii) EUK516 (5'-ACCAGACTTGCCCTCC-3') targeting the $18 \mathrm{~S}$ rRNA of $S$. cerevisiae in the artificially prepared samples, and of most Eukarya in real samples from Cultural Heritage goods [30].

More information about the applied probes can be found at probeBase [31].

\subsubsection{RNA-FISH analysis of yeast and bacterial isolates}

The starting point for optimizing the FISH procedure conditions was a modification of the methods previously developed by us $[25,27]$. These starting methods involved four sequential steps: fixation, hybridization, washing and analysis.

Fixation of the cells (section 2.1.1) was carried out with $5.0 \mathrm{~mL}$ of absolute ethanol for $1 \mathrm{~h}$ at room temperature[32]. The fixed cells were washed with PBS and preserved in $50 \% \mathrm{EtOH} / \mathrm{PBS}(\mathrm{v} / \mathrm{v})$ at $-20.0^{\circ} \mathrm{C}$, in centrifuge microtubes.

For hybridization, $5 \times 10^{5}$ yeast and $5 \times 10^{7}$ bacterial fixed cells were used for each assay. The fixed cells were washed with PBS and the volume of the resultant cellular suspension containing the cells was transferred to $1.5 \mathrm{~mL}$ microtubes and centrifuged. The hybridization buffer ( $20 \mu \mathrm{L}$ of queous solution, $0.9 \mathrm{M} \mathrm{NaCl}, 20 \mathrm{mM}$ Tris- $\mathrm{HCl}, 0.1 \%$ SDS, $\mathrm{pH}$ 7.2), was added to the pellet. The volume (0.1-2.0 $\mu \mathrm{L})$ of the correspondent RNA-FISH probe stock solution $(120 \mathrm{ng} / \mu \mathrm{L})$ was then added to each FISH sample. The probes used were EUB338 (for bacteria) and EUK516 (for yeast) labelled with Cy3, 6FAM or Cy5 (section 2.2.1). Both the blanks and FISH samples were incubated in a water-bath for $2 \mathrm{~h}$ at $46^{\circ} \mathrm{C}$ under continuous shaking. After centrifugation, the cells were washed with $25.0 \mu \mathrm{L}$ of pre-warmed hybridization buffer for $30 \mathrm{~min}$ in a water-bath maintaining the same conditions used for hybridization.

Finally, cells were pelleted by centrifugation, resuspended in $200.0 \mu \mathrm{L}$ of PBS and analyzed by epifluorescence microscopy and by flow cytometry.

The correspondent blanks, acting as controls for autofluorescence of the microbial cells, were subjected to standard FISH conditions without addition of the RNA-FISH 
probe. All the centrifugations cited in the experimental section were carried out for $5 \mathrm{~min}$ at $4500 \mathrm{rpm}$ and $4^{\circ} \mathrm{C}$ for $S$. cerevisiae cells, and for $15 \mathrm{~min}$ at $13000 \mathrm{rpm}$ for E. coli.

All the process was carried out under aseptic conditions and all the assays were performed in triplicate.

\subsubsection{RNA-FISH analysis of yeast-bacterial artificial consortia}

For analyzing the mixed suspensions of $E$. coli and $S$. cerevisiae cells by RNAFISH, yeast and bacterial isolates were fixed separately. Artificial consortia were prepared by mixing $5 \times 10^{7}$ and $5 \times 10^{5}$ fixed cells of E. coli and S. cerevisiae, respectively. The other steps for FISH analysis were performed as described in the section 2.2.2 using the following centrifugation conditions: $13000 \mathrm{rpm}$ for $15 \mathrm{~min}$ at $4^{\circ} \mathrm{C}$. Various experiments were carried out using the individual probes EUB338 or EUK516 labelled with Cy3 or 6-FAM and two different probe sets: EUB338-Cy3/EUK516-6-FAM and EUB338-6-FAM/EUK516-Cy3. They were prepared by mixing $1.0 \mu \mathrm{L}$ of the EUB338 and $0.25 \mu \mathrm{L}$ of the EUK516 fluorescently labeled probes.

\subsubsection{RNA-FISH analysis of real samples}

For each sample, the microbial suspension $(0.8 \mathrm{~mL})$ containing the recovered cells was centrifuged (15 min $13000 \mathrm{rpm})$. The supernatant was discarded and the cell pellet was fixed with absolute ethanol for $1 \mathrm{~h}(0.8 \mathrm{~mL})$. Thus, for hybridization, $0.4 \mathrm{~mL}$ of the cellular suspension were transferred to $1.5 \mathrm{~mL}$ microtubes (one for the FISH sample and other for the correspondent blank) and centrifuged. The subsequent steps were carried out as described for the artificial consortia (in section 2.2.3) but using exclusively the EUB338-Cy3/EUK516-6-FAM set of probes.

\subsubsection{Epifluorescence microscopy}

Fluorescence images were taken with a MoticamPRO 282B camera mounted on a BA410E Motic microscope coupled to a 100W Quartz Halogen Koehler illumination with intensity control and to an epi-attachment (EF-UPR-III) and a Power Supply Unit (MOTIC MXH-100). It was equipped with the Motic filter sets Cy3, (excitation (ex) D540/25x, dichroic mirror (dm) 565DCLP, and emission (em) D605/55m), FITC (ex D480/30x, dm 505DCLP, em D535/40m) and Cy5 (ex D436/20x, dm 455DCLP, em D480/40m). Images were recorded and analyze with the Motic Images Plus 2.0 ${ }^{\mathrm{LM}}$. 


\subsubsection{Flow cytometry}

Muse ${ }^{\circledR}$ Cell Analyzer and MuseSoft 1.4.0.0 software were used for flow cytometry analysis. Cells from the isolates, labelled with 6-FAM or Cy3, were analyzed for investigating the influence of the [probe]/[cell] ratios and cellular growth stage on the intensity of the probe-labelled cells. The Muse® Cell Analyzer is equipped with a 532$\mathrm{nm}$ green laser. Each sample was run in triplicate. For FISH samples 1000 events were acquired for each replicate, and for the blanks the maximal number acquired during the limited time for each run were analyzed $(>100)$. For each sample the fluorescence intensity was analyzed using the yellow photodiode detector (576/28). It was recorded on a gate that was first defined in a Fluorescence Intensity (FI)-versus-Forward Scatter density plot (considering blanks, FISH samples and non-treated samples).

\subsection{Autofluorescence tests}

The autofluorescence of the isolates, artificial consortia as well as microbial suspensions from real samples was tested by microscopic inspection of the cellular suspensions by epifluorescence microscopy using the Cy3, FITC and Cy5 filter sets before the analysis by RNA-FISH. No FISH signals were observed.

The autofluorescence of the fixed samples was also checked in order to avoid falsepositives, and they also did not show any detectable fluorescence.

\subsection{Complementary analysis of real samples for assessing the microbiota}

The analysis of the microsamples from the Escoural Cave by RNA-FISH was complemented with Scanning Electron Microscopy (SEM). They were done directly as collected or coated with Au-Pd (Quorum Q150R ES) during $300 \mathrm{~s}$, at $25 \mathrm{~mA}$, and observed in a HITACHI S-3700N variable pressure scanning electron microscope (VPSEM) with accelerating voltage of $9-10 \mathrm{kV}$. For determining the reliability of the RNAFISH protocol to assess the microbiota inhabiting $\mathrm{CH}$ materials, results were also compared with further analyses involving culture-dependent and culture-independent approaches which were developed in another publication [33].

\section{Results and discussion}

It is well known that for improving or adapting RNA-FISH technique, new conditions needs to be carefully investigated and selected in order to avoid the occurrence of false positive or negatives [11,34,35].

In $\mathrm{CH}$ microbiology applications, selecting the conditions for maximizing the fluorescence intensities obtained by RNA-FISH, as the [probe]/[cell] ratio or the 
fluorophores labeling the probes[34], is crucial. Many factors can hamper the detection of the probe-conferred fluorescence (e.g. background fluorescence due to the presence of autofluorescent microorganisms or particular of the $\mathrm{CH}$ material) $[11,13,14,36]$. However, the fluorochromes'selection is only based on avoiding the interference with autofluorescent material, from the support or from other microorganisms [6,14]. No extra criteria have been reported in the literature justifying it, even though, the choice of proper fluorochromes is a key point for the success on the application of RNA-FISH [34].

It is also well known that in monument surfaces most of microorganisms can be in a dormant state containing a low number of rRNA copies and that the RNA-FISH signals are strongly dependent on the cellular RNA content [29,35].

In this way, the effect of various factors influencing the RNA-FISH results (fluorochromes' selection and [probe]/[cell] ratio, growth stage of the cells) were firstly investigated in pure culture experiments in order to establish the optimal conditions for detecting each type of microorganism.

\subsection{Factors influencing the results: Pure-culture experiments}

With this aim, several experiments were carried out: i) maintaining constant the concentration of fixed cells that were hybridized and varying the concentrations of the probes; ii) using the probes EUB338 and EUK516 targeted with various fluorophores: Cy3, Cy5 or 6-FAM; and iii) analyzing cells in various growth stages, with different RNA contents.

Independently of the [probe]/[cell] ratio, intense FISH signals were obtained for both microorganisms when the specific probes labelled with Cy3 or 6-FAM were used (in Online Resource 1, Tables S1 and S2). The microphotographs captured for the minimal and maximal [probe]/[cell] ratios are summarized in tables 1 and 2 . The signals were more intense for $\mathrm{Cy} 3$ than for 6-FAM, but both fluorochromes allowed the detection of bacteria and yeast cells. However, the EUB338-Cy5 probe did not allowed to detect the bacteria cells at any of the concentrations tested and the EUK516-Cy5 only allowed to visualize yeast cells with extremely low intensity (too low compared to those obtained when using the other two fluorophores). Rapid photobleaching of Cy5 was also observed during microscopic inspection (in few seconds of light exposure the fluorescent signals disappeared). These results are in accordance with the lack of signal that has been previously referred by other authors when applying Cy5 for targeting microorganisms using RNA-FISH probes (ARCH915-Cy5 or EUB338-Cy5) in $\mathrm{CH}$ studies [6,11]. However, in contrast with the hypothesis raised in those works to explain it (low RNA 
content or low probe permeability), the results obtained in this work pointed out to the low fluorescence intensity associated to the fluorochrome and to its rapid photobleaching as possible reasons for the absence of fluorescent signals. Conversely, good RNA-FISH signals have been previously obtained by other authors using the same probe EUB338Cy5 and paraformaldehyde as fixative for detecting bacteria in enriched samples from $\mathrm{CH}$ [36]. This evidence that the results strongly depend of the protocol used and highlights again the importance of investigating the factors that can affect the signals obtained for each protocol, in order to avoid misleading conclusions.

The behavior described above was found for all the growth stages investigated (Table 3): Cy3 and 6-FAM labelled probes allowed to detect the yeast and bacteria cells, whereas Cy5-target probes yields none or extremely weak fluorescent signals.

Thus, the probes labelled with Cy5 were not considered in the next experiments and they were also excluded from consideration for this in-suspension RNA-FISH method.

The cells labeled with Cy3 and 6-FAM were also analyzed by flow cytometry. In accordance with the microscopic observations, the cells of both microorganisms were satisfactorily detected, independently of the growth phase and the [probe]/[cell] ratio (Online Resource 1,Table S3). Considering this, the RNA-FISH method used is mainly limited by the analytical capacity of the flow cytometry and microscopic techniques. Attending to the conditions used for RNA-FISH, the minimal number of cells required for their detection in a sample are around 500 cells.

In the basis of the results obtained in the single staining assays the best probe combination to be used for simultaneous analysis of yeast and bacteria cells seems to be EUB338-Cy3/EUK516-6-FAM. On one hand, the microscopic analysis revealed that: i) for the bacteria cells (smaller and more difficult to detect than yeast) the EUB338-Cy3 produced the most intense fluorescent signals from those tested (Table 1); and ii) intense fluorescent yeast cells were observed using EUK516-6-FAM for targeting them (Table 2 ). Also, the flow cytometry analysis of $S$. cerevisiae and E. coli isolates stained with EUK516-6-FAM and EUB338-Cy3, respectively, suggested the possibility of distinguishing the stained cells of both microorganisms (Fig.1). This preliminary results pointed out the possibility of using this in-suspension RNA-FISH protocol in the future for its application in combination with flow cytometry (Flow-FISH) for analyzing microbial communities composed by this kind of microorganisms

In the best of our knowledge this combination of probes has not been applied before using this RNA-FISH dual staining protocol. In fact, whereas $\mathrm{Cy} 3$ and $\mathrm{Cy} 5$ have been 
previously used for targeting microbial colonizers of $\mathrm{CH}$ goods, 6-FAM has not previously employed in this field. Instead, FITC a fluorochrome with similar spectral properties but more susceptible to photobleaching has been usually employed $[5,10,19,37]$.

\subsection{Simultaneous analysis of yeast and bacteria cells in mixed suspensions: Dual staining}

On the basis of the results obtained in the pure culture assays, for the simultaneous analysis of yeast and bacteria, only the combinations of EUK516 and EUB338 labeled with Cy3 or 6-FAM were tested for analyzing yeast and bacteria simultaneously.

To confirm that the probes mix EUK516-6-FAM/EUB338-Cy3 is a better alternative than the use of EUK516-Cy3/EUB338-6-FAM, the following experiments were carried out using artificial consortia:

i) both probes mixes were tested in independent assays EUK516-6-FAM/EUB338Cy3 and EUK516-Cy3/EUB338-6-FAM and analyzed with the filter sets specific for each fluorochrome: FTIC and Cy3 filter sets;

ii) all the probes cited above were also applied in individual FISH assays and analyzed with both filter sets in order to ensure that the FISH protocol applied allows specific detection of the target cells and avoid false-positives. It means that the RNAFISH method does not exhibit cross-fluorescence interference;

iii) the correspondent blanks for all the FISH assays mentioned were also prepared and analyzed showing no detectable fluorescence (data not shown).

The results confirmed that the use of EUK516-6-FAM/EUB338-Cy3 combination (Table 4) is the best alternative from both tested as allows to detect specifically the target cells in the correspondent filter set, without any interference (Fig 2). The probe-conferred fluorescence of the $\mathrm{Cy} 3$-targeted bacteria cells is intense and the non-specific fluorescence detected in the $\mathrm{Cy} 3$ filter for the 6-FAM-targeted yeast cells contributed exclusively to the background fluorescence. This is in agreement with the results of the individual probe assays, that showed no signals for the bacteria targeted with EUB338Cy3 using the FTIC filter but weak signals for the yeast targeted with EUK516-6FAM using the $\mathrm{Cy} 3$ filter (Table 4). This is due to the spectral properties of Cy3 and 6-FAM dyes, Cy3 does not emit light under the FITC filter. Although 6-FAM emits light under the Cy3 filter, albeit of low intensity.

The microphotographs obtained for the EUK516-Cy3/EUB338-6-FAM mix revealed that this combination of probes do not allow the detection of both microorganisms. 
Intense fluorescent yeast cells were captured (Table 4). However, weak fluorescent bacteria cells were detected, the fluorescence were as low as it was impossible to capture them. These results were corroborated by the analysis of the artificial consortium with the individual probes (Table 4).

Thus, an in-suspension RNA-FISH protocol for detecting yeast and bacteria simultaneously have been developed. It involves the use of the EUB338-Cy3/EUK5166-FAM probes. It was validated using artificial E. coli -S. cerevisiae consortium (Fig. 2).

To our knowledge this is the first work focused on the development and application of RNA-FISH in-suspension for simultaneous analysis of microorganisms from different kingdoms, Eukarya and Eubacteria, in $\mathrm{CH}$ samples.

\subsection{Application of the RNA-FISH method for simultaneous analysis of yeast and bacteria in real samples}

The in-suspension RNA-FISH method previously validated by using artificial consortia was used for simultaneously assessment of the presence of yeast and bacteria in real samples of the Palaeolithic site of the cave of Escoural (Alentejo region, Portugal). It is the only cavity discovered in Portugal where Upper Paleolithic rock art (35000$10000 \mathrm{BP}$ ) has been identified [38]. It hosts numerous paintings and engravings, most of which are altered due to weathering and environmental conditions. Microsamples from biological breaches spotted inside the cave (Fig. 3a and 3b) were carried out to study the metabolically active microbial proliferating inside the cave. The analysis by Scanning Electron Microscopy (SEM) of GdE 1 and GdE 2 microsamples confirmed the presence of biofilms covering a significant area of the microfragments as well as of microbial contaminants (Fig. 3c and 3d). Among others, cells with reduced dimension were detected, which can be indicative of the presence of bacterial cells (Fig. 3c).

The microbial cells extracted from the two microfragments from the Escoural Cave, GdE1 and GdE2, were analyzed by the RNA-FISH method as described in the methodology. None autofluorescence signals were observed for the blanks. This notably facilitated the analysis of the results obtained by RNA-FISH. By applying the EUB338Cy3/EUK516-6-FAM mix, only intense orange signals were obtained by microscopic inspection. This suggested the presence of active Eubacteria living in the Escoural Cave and the absence of yeast (Fig. 3e and 3f). These preliminary results were supported by those obtained in a complete study about the biodeteriogenic activity of microbial biofilms' communities of Escoural Cave, also carried out in our research group [33]. The results obtained in this work, by both culture-dependent and NGS approaches, enabled to 
confirm that the Escoural Cave was: i) predominantly colonized by Prokaryote cells, representing around $90 \%$ of the microbial community, that are also active microorganisms; ii) not colonized by a detectable quantity of yeast [33].

Thus, it can be concluded that satisfactory preliminary results were obtained by applying the improved dual staining RNA-FISH protocol. This suggest its applicability for analyzing $\mathrm{CH}$ microcolonizers, whereas more efforts are required to validate the protocol for its use in real samples, particularly for stablishing the optimal storage conditions.

\section{Conclusions}

The results of this work allowed to highlight the importance of a careful selection of the conditions used for avoiding the occurrence of false positive or negatives when applying RNA-FISH. They evidenced also that particular attention must be focused on the selection of the appropriate fluorochromes for labelling the probes when the protocols are being adapted or modified.

Microscopic analysis of the bacterial and yeast isolates with the in suspension RNA-FISH protocol revealed the impossibility to use Cy5 for labelling EUB338 or EUK516 in the attempt of cells detection.

Flow-cytometry analysis of the single stained isolates, points out the possibility of applying the in-suspension RNA-FISH protocol for analyzing yeast and bacteria stained with EUB338-Cy3 and EUK516-6-FAM.

The main output of this work was a dual staining in-suspension RNA-FISH protocol that allow simultaneous detection of yeast and bacteria using EUB338Cy3/EUK516-6-FAM probe set. The EUB338-Cy3/EUK516-6-FAM gave notably better results than the EUB338-6-FAM/EUK516-Cy3 combination. The protocol was validated for simultaneous analysis of yeast and bacterial cells using artificial consortia and have also shown good preliminary results in the analysis of $\mathrm{CH}$ samples. Whereas more work is required to evaluate the potentials and limitations of the dual staining in-suspension RNA-FISH method on analyzing real samples, the results obtained are promising and open the door for the future implementation of Flow-FISH technique for investigating the biodiversity hosted on $\mathrm{CH}$ assets. This encourages us to continuous working for the RNAFISH in-suspension protocol and Flow-FISH technique to become practical tools for investigating the biodeterioration of artworks. 



\section{References}

1. P. Sanmartín, A. DeAraujo, and A. Vasanthakumar, Microb. Ecol. 1 (2016).

2. A. Mihajlovski, D. Seyer, H. Benamara, F. Bousta, and P. Di Martino, Ann. Microbiol. 65, 1243 (2015).

3. J. L. Boutaine, Phys. Tech. Study Art, Archaeol. Cult. Herit. 1, 1 (2006).

4. C. Schabereiter-Gurtner, G. Piñar, W. Lubitz, and S. Rölleke, J. Microbiol. Methods 45, 77 (2001).

5. C. Urzí and P. Albertano, in Methods Enzymol., edited by J. D. Ron (Academic Press, 2001), pp. 340-355.

6. G. Piñar and W. Lubitz, in Http://Www.Itam.Cas.Cz/ Arcchip/Ariadne_8.Shtml (2004), p. 12.

7. S. Baskar, R. Baskar, L. Mauclaire, and J. A. McKenzie, Curr. Sci. 90, 58 (2006).

8. F. Cappitelli, P. Principi, and C. Sorlini, Trends Biotechnol. 24, 350 (2006).

9. F. Cappitelli, P. Principi, R. Pedrazzani, L. Toniolo, and C. Sorlini, Sci. Total Environ. 385, 172 (2007).

10. A. Santos, A. Cerrada, S. García, M. San Andrés, C. Abrusci, and D. Marquina, Microb. Ecol. 58, 692 (2009).

11. E. Müller, U. Drewello, R. Drewello, R. Weißmann, and S. Wuertz, J. Cult. Herit. 2, 31 (2001).

12. A. Polo, F. Cappitelli, L. Brusetti, P. Principi, F. Villa, L. Giacomucci, G. Ranalli, and C. Sorlini, Microb. Ecol. 60, 1 (2010).

13. D. B. Meisinger, J. Zimmermann, W. Ludwig, K.-H. Schleifer, G. Wanner, M. Schmid, P. C. Bennett, A. S. Engel, and N. M. Lee, Environ. Microbiol. 9, 1523 (2007). 14. K. Sterflinger and M. Hain, Stud. Mycol. 1999, 23 (1999).

15. S. Muller and G. Nebe-von-Caron, FEMS Microbiol Rev 34, 554 (2010).

16. C. Urzì, in Herit. Microbiol. Sci. Microbes, Monum. Marit. Mater., edited by E. May, M. Jones, and J. Mitchell (Royal Society of Chemistry (Great Britain), Cambridge, 2008), pp. $143-150$.

17. K. Sterflinger, W. E. Krumbein, and A. Schvviertz, Int. Microbiol. 1, 217 (1998).

18. C. Urzì, F. De Leo, P. Donato, and V. La Cono, in Art, Biol. Conserv. Biodeterior. Work. Art, edited by R. J. Koestler and M. M. Art (Metropolitan Museum of Art, NY, 2003), pp. 317-325.

19. C. Urzì, V. La Cono, and E. Stackebrandt, Environ. Microbiol. 6, 678 (2004).

20. F. Villa, F. Cappitelli, P. Principi, A. Polo, and C. Sorlini, Lett. Appl. Microbiol. 48, 
234 (2009).

21. V. La Cono and C. Urzì, J. Microbiol. Methods 55, 65 (2003).

22. F. De Leo and C. Urzi, Fungi from Differ. Substrates 144 (2015).

23. R. I. Amann, L. Krumholz, and D. A. Stahl, J Bacteriol 172, 762 (1990).

24. C. Urzì and F. De Leo, J. Microbiol. Methods 44, 1 (2001).

25. R. Vieira, P. Nunes, S. Martins, M. González, T. Rosado, A. Pereira, A. Candeias, and A. T. Caldeira, in Sci. Technol. Cult. Herit., edited by A. Rogerio-Candelera (Taylor \& Francis Group, London, 2014), pp. 257-262.

26. R. Vieira, M. González-Pérez, A. Pereira, A. Candeias, and A. T. Caldeira, Conserv. Património 23, 71 (2016).

27. M. González, R. Vieira, P. Nunes, T. Rosado, S. Martins, A. Candeias, A. Pereira, and A. T. Caldeira, E-Conservation J. 44 (2014).

28. Y. Williams, S. Byrne, M. Baschir, A. Davies, A. Whelan, Y. Gun'ko, D. Kelleher, and Y. Volkov, J. Microsc. 232, 91 (2008).

29. E. Nettmann, A. Fröhling, K. Heeg, M. Klocke, O. Schlüter, and J. Mumme, BMC Microbiol. 13, 278 (2013).

30. R. Amann and B. M. Fuchs, Nat. Rev. Microbiol. 6, 339 (2008).

31. D. Greuter, A. Loy, M. Horn, and T. Rattei, Nucleic Acids Res. 44, D586 (2016).

32. R. I. Amann, W. Ludwig, and K. H. Schleifer, Microbiol. Rev. 59, 143 (1995).

33. G. Mauran, T. Rosado, C. Salvador, N. Schiavon, J. Mirão, A. T. Caldeira, and A. Candeias, Int. Biodeterior. Biodegradation In press (2016).

34. T. Bouvier and P. A. Del Giorgio, FEMS Microbiol. Ecol. 44, 3 (2003).

35. G. Wallner, R. Amann, and W. Beisker, Cytometry 14, 136 (1993).

36. G. Piñar, C. Ramos, S. Rölleke, C. Schabereiter-Gurtner, D. Vybiral, W. Lubitz, and E. B. M. Denner, Appl. Environ. Microbiol. 67, 4891 (2001).

37. C. Urzì, V. La Cono, F. De Leo, and P. Donato, Mol. Biol. Cult. Heritage. Lisse, Netherlands, Balkema Publ. 55 (2003).

38. F. dos Santos, O Arqueol. Port. 2, 5 (1964). 


\section{Tables}

Table 1 RNA-FISH microphotographs of S. cerevisiae cells in stationary phase stained with EUK516-Cy3, EUK516-Cy5 and EUK516-6-FAM probes for the minimal and maximal [probe]/[cell] ratio tested.

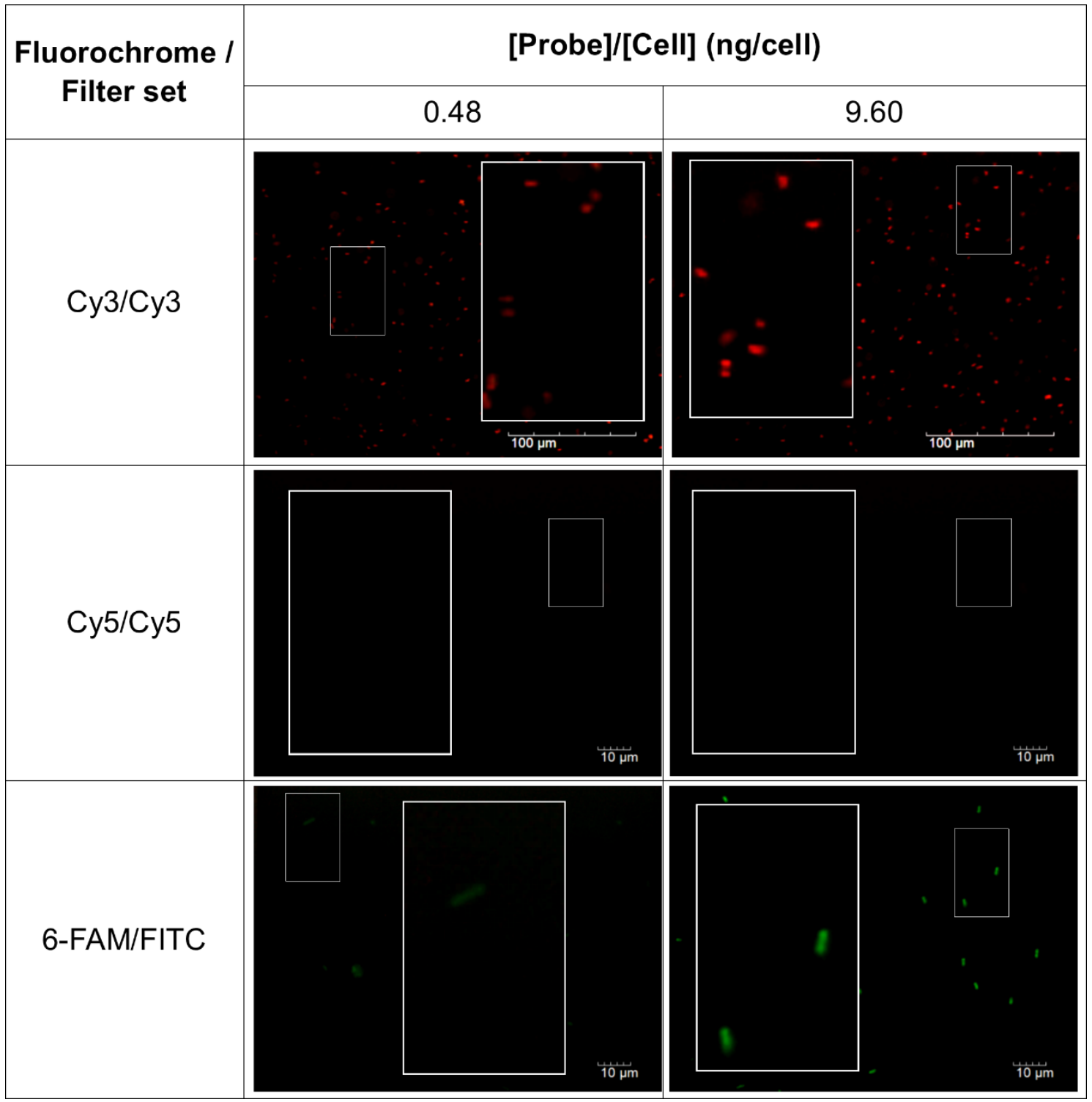


Table 2 RNA-FISH microphotographs of $E$. coli cells in exponential phase stained with EUB338-Cy3, EUB338-Cy5 and EUB338-6-FAM probes for the minimal and maximal [probe]/[cell] ratio tested

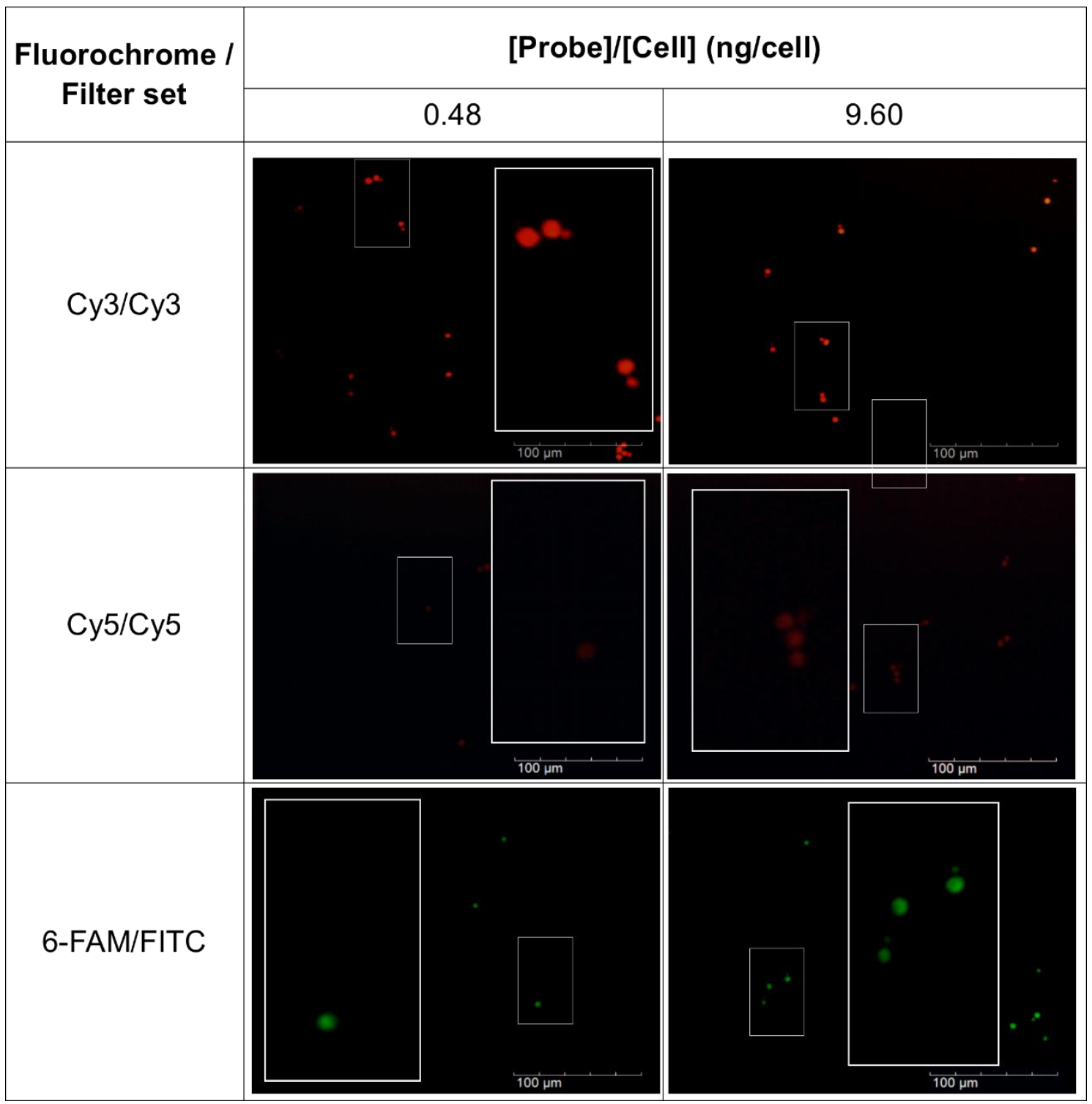


Table 3 Influence of the cellular growth phase and of the fluorochrome labeling the probe on the FISH results. FISH microphotographs from the analysis of E. coli and $S$. cerevisiae cells in latency, exponential and stationary growth stages using the EUB338 and the EUK516 oligonucleotides labeled with -Cy3, -Cy5 or-6-FAM at the 5' end

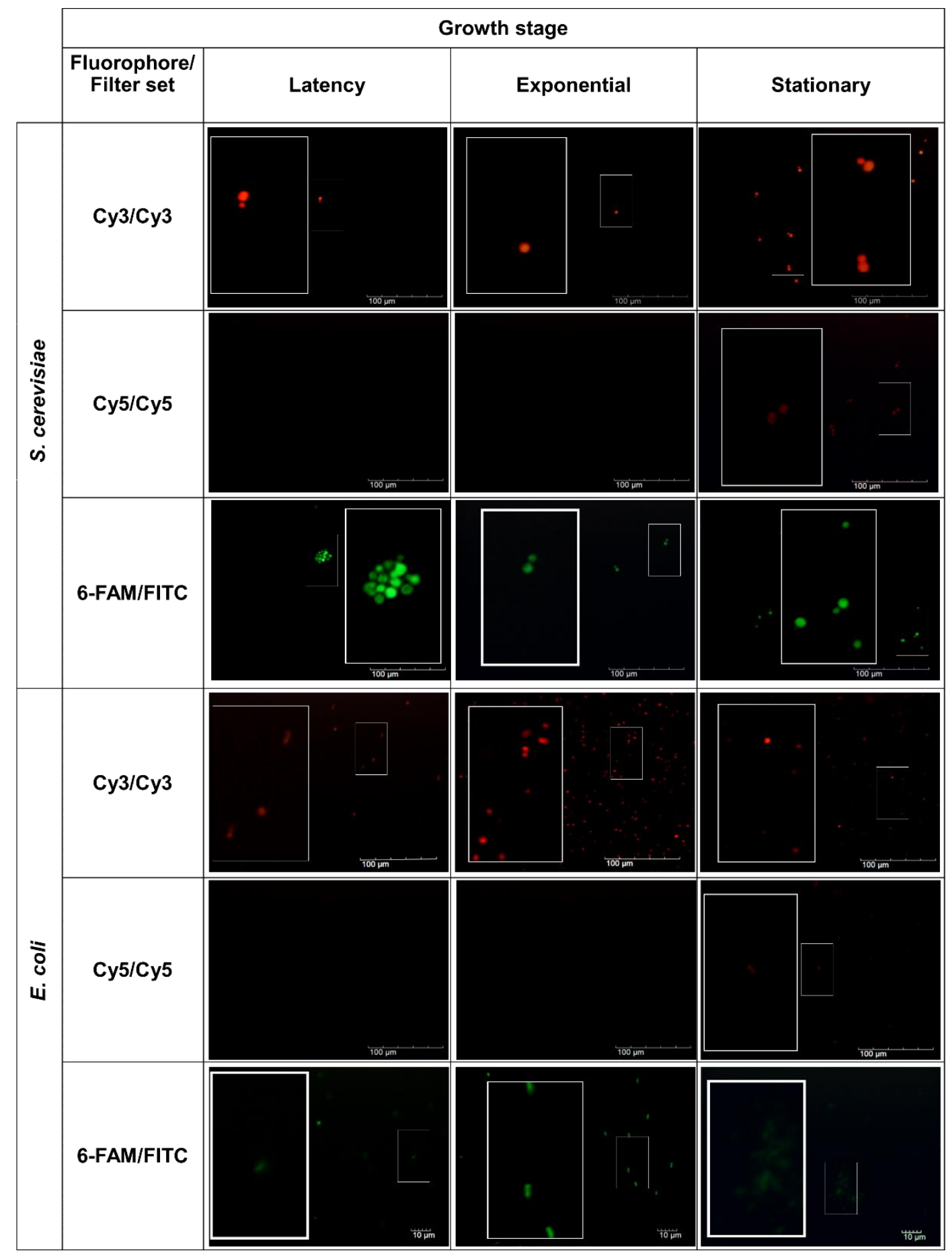


Table 4 FISH microphotographs from the analysis of artificial consortia of E. coli and S. cerevisiae using the EUB338 and EUK516 probes labelled with Cy3 or 6-FAM in assays performed with single probes for single staining or probe sets for dual staining 


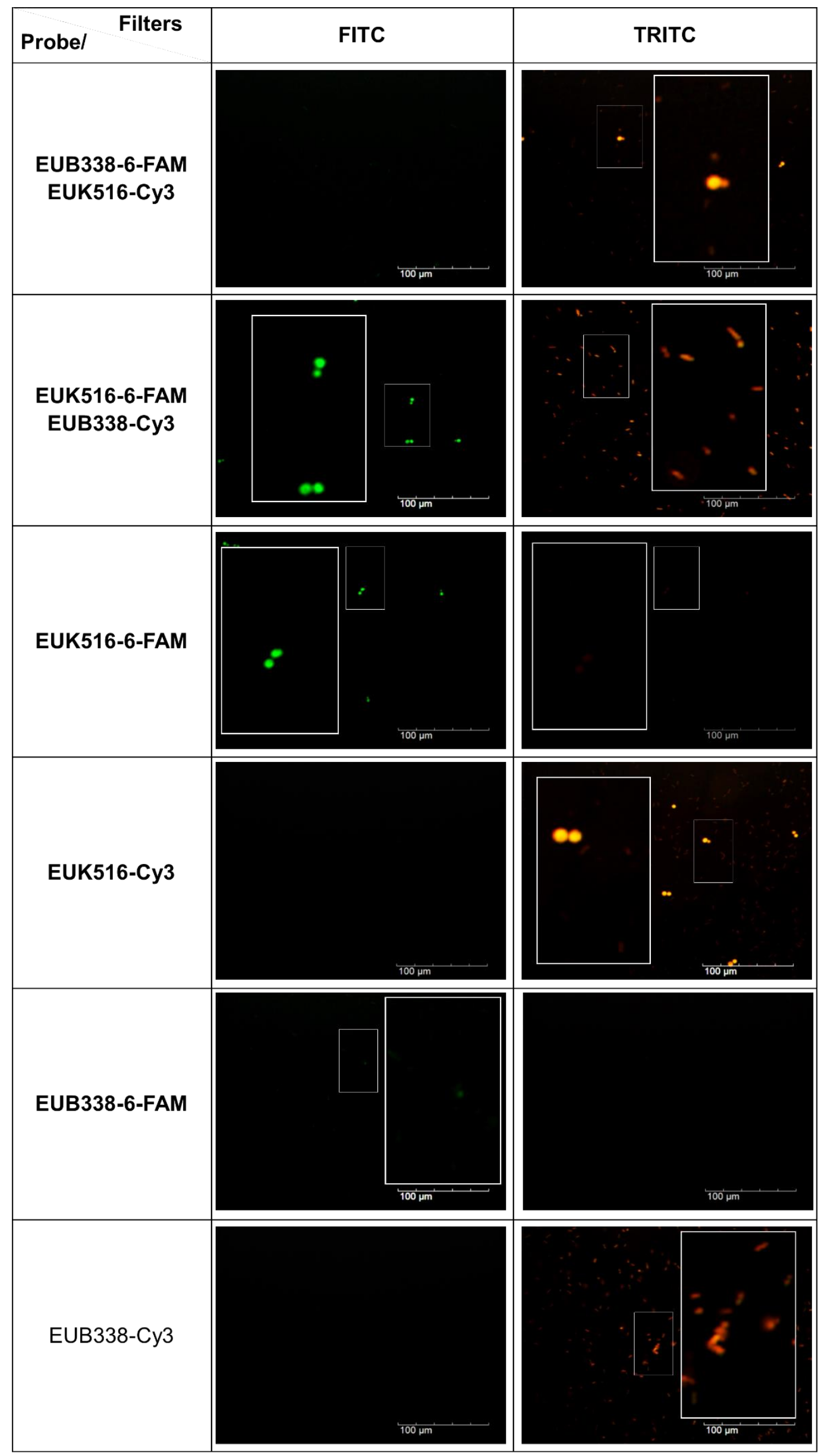




\section{Figures}
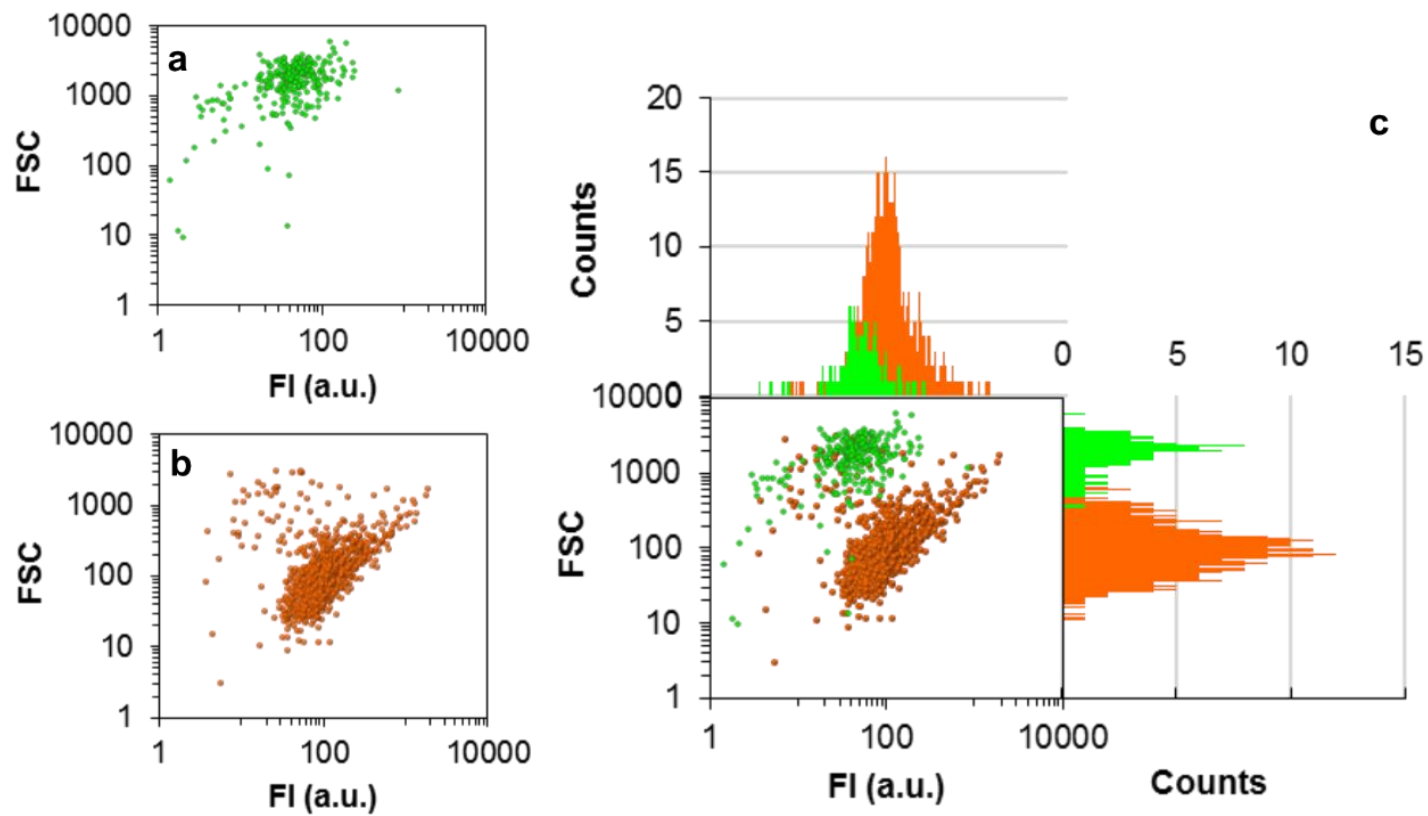

Fig. 1 Flow cytometry results. Fluorescence Intensity (FI)/Forward Scattering (FSC) density plots, for the RNA-FISH single staining assays using isolates of $E$. coli (a) and $S$. cerevisiae (b) cells in exponential phase using EUB338-Cy3 (orange) and EUK516-6FAM (green) probes, respectively. Comparison of the flow cytometry results (c): merged Fluorescence Intensity (FI)/Forward Scattering (FSC) density plots, one-parameter histograms of the FI and FSC.

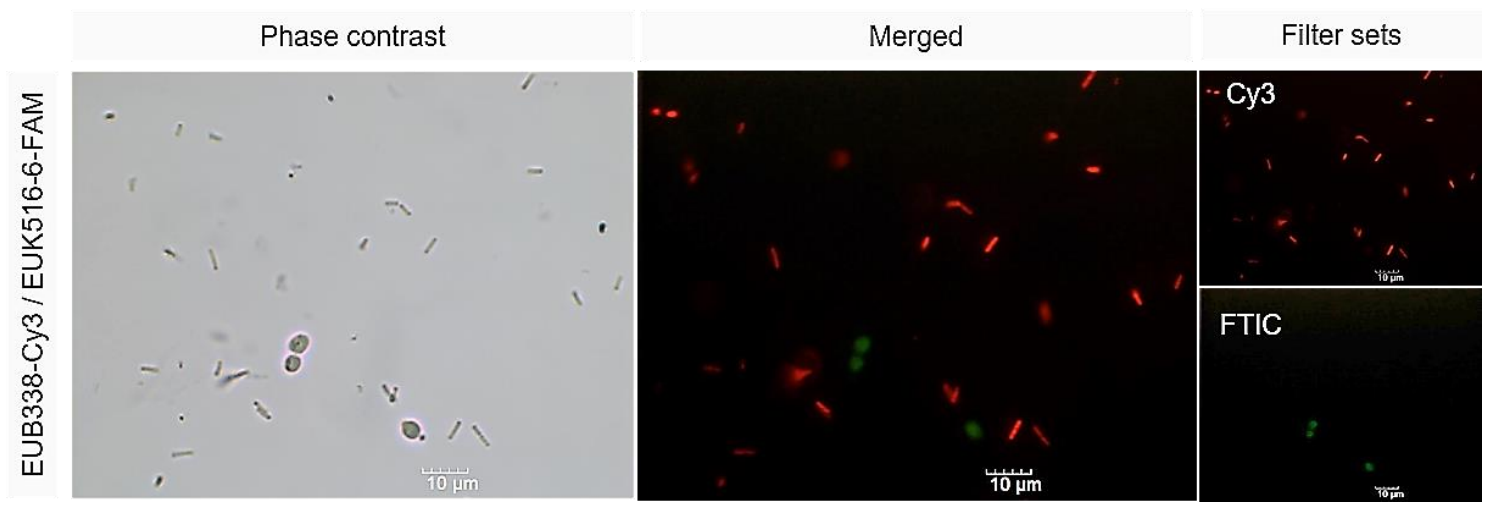

Fig. 2 Epifluorescence micrographs of artificial consortium of S. cerevisiae and $E$. coli cells obtained using the optimized in-suspension RNA-FISH protocols with the EUK516-6-FAM/EUB338-Cy3 mix. Phase contrast and merged fluorescence in situ hybridization (FISH) image of the signals of E. coli using the Cy3 filter (orange) and $S$. cerevisiae (green) using the FITC filter 


\section{GdE 1}

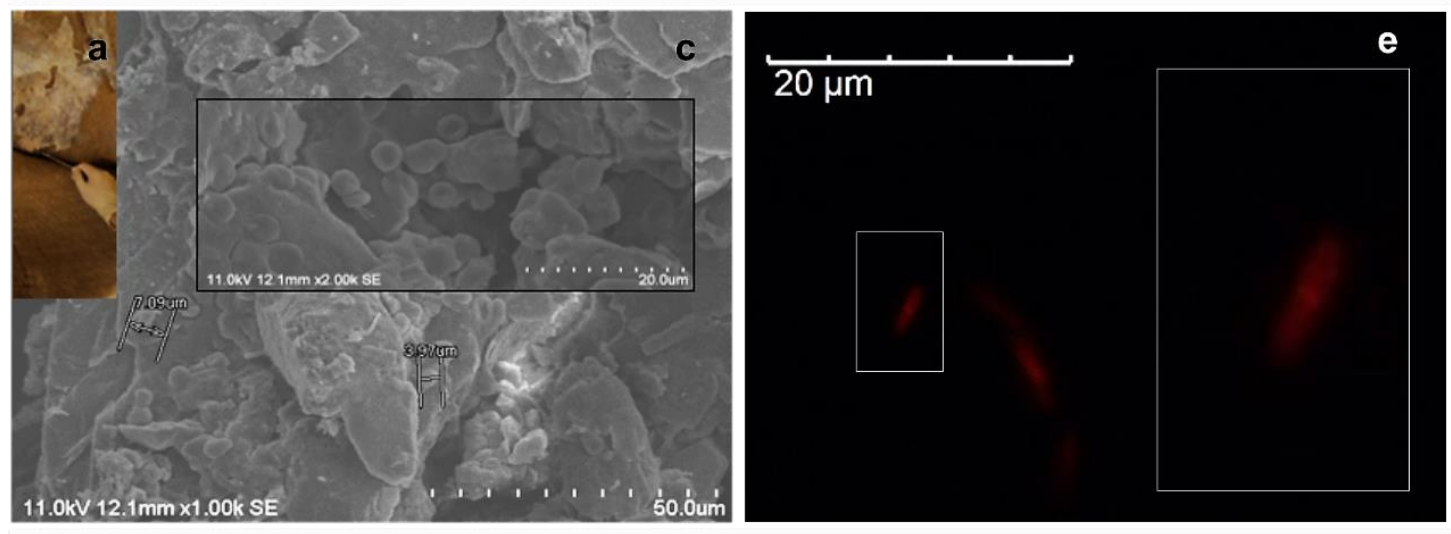

GdE 2

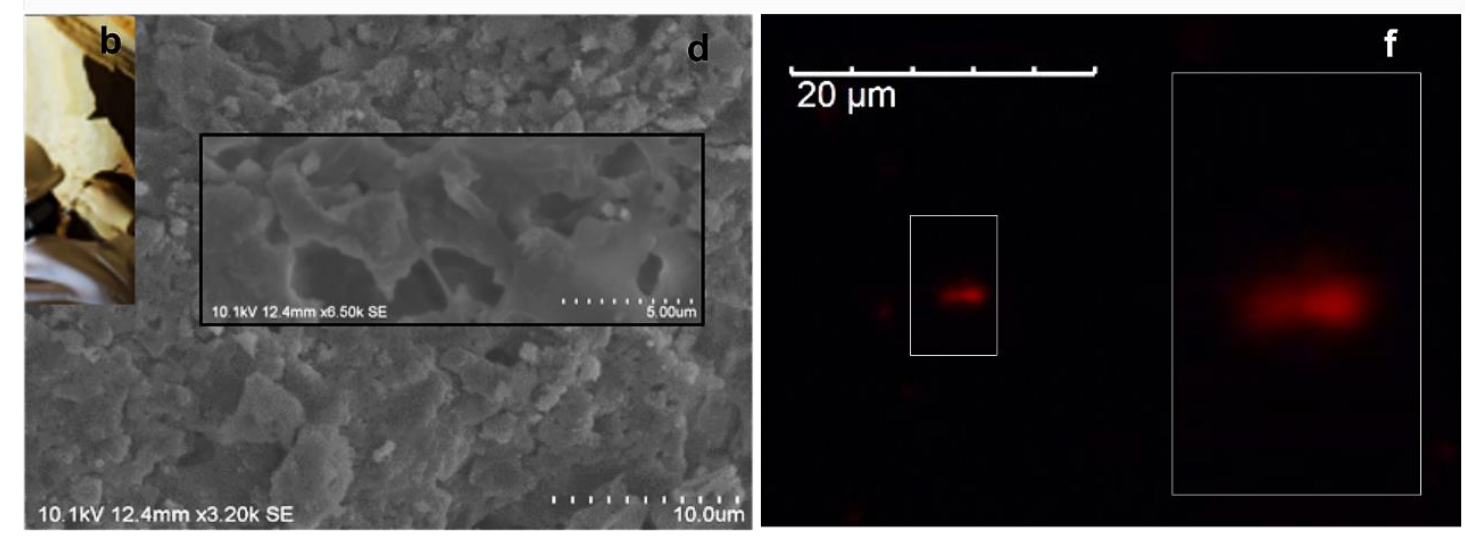

Fig. 3 Analysis of rock microfragments GdE 1 and GdE from Escoural Cave (a, b), by: i) SEM micrographs (c,d) and ii) the dual staining RNA-FISH protocol developed in this work, using the EUB338-Cy3/EUK516-6-FAM mix- (e, f); 